\title{
AOR
}

Selected Papers of \#AolR2020:

The $21^{\text {st }}$ Annual Conference of the

Association of Internet Researchers

Virtual Event / 27-31 October 2020

\section{THE DEAD SPEAK: BIG DATA AND DIGITALLY MEDIATED DEATH}

\author{
Justin Grandinetti \\ University of North Carolina Charlotte \\ Tyler DeAtley \\ North Carolina State University \\ Jeffery Bruinsma \\ North Carolina State University

\section{Introductory Statement}

In the following panel, we add to scholarly challenges regarding the binary distinction between life and death by examining new strategies of making productive the data of the dead. Digital media and tactics of big data collection, storage, and processing blur the boundaries of human lifecycles, allowing the individual to exist as a productive part of sociotechnical apparatuses long after their corporeal demise.

In colloquial discussions, life and death are often conceptualized as dichotomous; yet, complicating this distinction is a conceptualization of life and death as coextensive, as human and nonhuman bodies exist in a constant state of becoming (Ingraham \& Rowland, 2016). Clarified by Rosi Braidotti (2010), life itself is a relentlessly generative force that requires interrogation of human and nonhuman entanglement. The imbrication of users, technologies, data collection practices, infrastructures, platforms, digital flows, etc. contributes to an erosion of the bounded human being, giving way to a more productive, nonbinary distinction "between same and other, between different categories of living beings, and ultimately between life and death" (Braidotti, 2010, p. 209). Put more simply, the origins and endpoints of corporeal life and death cannot account shifting forms of existence enabled by compositions of users, data, and digital media. Or, even more succinctly, "death is overrated" (Braidotti, 2010, p. 212).

Reconceptualizing life and death requires attention to how media are relevant to organic and inorganic states of becoming. Beginning with the inception of the telegraph, media allowed new forms of simultaneity, liveness, and co-presence that has been framed as ghostly and haunted (Sconce, 2009). Explained by Dorthe Christensen and Kjetil Sandvik (2016), "Media enable us to establish, maintain and develop relations with the dead without being present in the same space-time continuum" (p. 1). Accounting for 
contemporary social media practices, scholarship has examined how platforms enable changing discourses about birth and death (Leaver \& Highfield, 2016), including how social media memorialization has complicated the ethics and cultural understanding of death (Wright, 2014). In spaces of pervasive web connectivity, the social media subject is required to use technology to maintain the boundaries of a self, even when the distinctions between humans and data, life and death are being gradually erased (Bollmer, 2016). Adding to the important work of these discussions, we endeavor to rethink "the temporality of the subject such that the human is no longer constrained by death, but rather an evolving biophysical form" (Ingraham \& Rowland, 2016, p. 10). We contend that the continued relevance of deceased user's data necessitates reconsideration of the power dynamics, monetization, and ethics of digitally-mediated lifecycles.

In this panel, we turn these insights toward an examination of death and big dataspecifically, our presentations on digital data and death focus on the topics of subjectivation, consent and privacy, and commodification. Reanimator: Haunted Data, Streaming Media, and Subjectivity examines the process of subjectivation taking into account the haunted data and digital afterlives of streaming media. Subjectivation is often conceptualized as producing a docile human subject that functions toward machines of capitalism, war, and bureaucracy; however, compositions of big data, platforms, infrastructures, and algorithms offer the possibility of a productive sociotechnical economic subject unbounded from the human body. Grief by the Byte: Constructions of Data Consent, Privacy, and Stability in Griefbots interrogates the data practices and ethics related to the creation of chatbots from the data of deceased individuals. While these so-called griefbots are framed as helpful to those grieving a lost loved one, there remain questions about consent and privacy that accompany these interactions. Finally, What is Dead May Never Die: The Commodification of Death in Social Media Advertising studies how user data maintains economic value after death via networks designed to surveil, collect, and commodify the immaterial labor of the dead. The implications of an economic future largely influenced by the data of the deceased are troublesome, especially if the available data on various digital platforms eventually belongs mostly to the dead. Taken together, these presentations add to an exigent focus on the relationship between life, death, digital media, and big data.

\section{References}

Bollmer, G. (2016). Inhuman networks: social media and the archaeology of connection. New York: Bloomsbury Academic.

Braidotti, R. (2010). The Politics of "Life Itself" and New Ways of Dying. In New Materialisms: Ontology, Agency, and Politics. Durham: Duke University Press.

Christensen, D. R., \& Sandvik, K. (2016). Mediating and remediating death. Abingdon, Oxon: Routledge. 
Ingraham, C., \& Rowland, A. (2016). Performing Imperceptibility: Google Street View and the Tableau Vivant. Surveillance \& Society, 14(2), 211-226.

Leaver, T., \& Highfield, T. (2016). Visualising the ends of identity: pre-birth and postdeath on Instagram. Information, Communication \& Society, 21(1), 30-45.

Sconce, J. (2009). Haunted media: electronic presence from telegraphy to television. Durham: Duke University Press.

Wright, N. (2014). Death and the Internet: The implications of the digital afterlife. First Monday, 19(6).

REAMINATOR: HAUNTED DATA, STREAMING MEDIA, AND SUBEJCTIVITY

Justin Grandinetti

University of North Carolina Charlotte

In the era of big data, information circulates long after the death of individual users in what has been termed digital afterlives (Wright, 2014), data afterlives (Fuller, 2009), and haunted data (Blackwell, 2019). For example, Öhman and Watson (2019) project that there will be over 1.4 billion Facebook profiles of deceased users by the year 2100, leading to questions of the dead overtaking the living online. While much of the discourse surrounding the data of the dead has centered around social media platforms, these haunted traces exist in ever-expanding forms that include streaming media. Streaming platforms utilize data metrics that include what users watch, the duration of viewing, and location and device data in the creation and suggestion of content (Saltzman, 2018). Magnetically inscribed on servers, these data metrics "bear traces of human, material, technical, symbolic, and imaginary histories" (Blackman, 2019, p. 166) that function toward the systematic digital monitoring of people or groups to regulate or govern behavior (Esposti, 2014). Put another way, data traces-at once living and nonliving, human and technical-are an essential element in machinic compositions that channel affective intensities, produce subjects, and enable agency. Consequently, this use of haunted data challenges not only the distinction between life and death, but also the notion of the bounded, corporeal human subject.

This presentation addresses the process of subjectivation taking into account the haunted data and digital afterlives of streaming media. Scholarship has examined the production of the subject as socially bounded and formed via ideology (Althusser, 1971), by discursive formations (Foucault, 1969), or by collective assemblages (Deleuze \& Guattari, 1983 [1972]). Despite different conceptualizations of how the subject is formed, subjectivation often functions toward the engineering of "docile subjects as functional components of the sociotechnical megamachines of war, bureaucracy, and/or capital" (Wiley \& Elam, 2018, p. 222). In considering big data and networked connectivity, however, Bollmer (2016) contends that "Social media produce the human as a 'posthuman' subject...through a deeply ingrained and ultimately 
quotidian belief that it is in human nature to connect and circulate flows of information and capital" (p. 5). Social media, then, is part of a transformed governmentality, in which the "nodal citizen" is expected to engage in and internalize proper conduct that includes connecting and maintaining flows while simultaneously managing the definitions and limits of their own life (Bollmer, 2016, p.7; 119). Not only does streaming media contribute to these forms of ubiquitous user data collection, but these platforms are increasingly oriented toward social interactivity (Spilker, Ask, \& Hansen, 2018). For example, global users now stream content across a variety of platforms: the televisual and cinematic content of Netflix, Hulu, and iQiyi; social media streaming through Facebook, Instagram, and YY; and mobile-social streaming via TikTok, Perisciope, and Quibi. Streaming, accordingly, represents a multitude of data collection that includes countless data metrics derived from user-platform interactions as well as flows of audiovisual inscription via user-uploaded content.

To examine the relationship between streaming media, death, and subjectivity, I draw upon Wiley and Elam's (2018) synthetic subjectivation and Blackman's (2019) hauntology. In a re-reading of Deleuze and Guattari's three syntheses of subjectivation, Wiley and Elam (2018) position synthetic subjectivation "as a way to conceptualize subject formation as grounded in compositions of heterogeneous elements, and not in humans or hominid organisms" (p. 207). To this end, Wiley and Elam (2018) eschew the quotidian signifying distinction between human and technology to consider the composite body of natural-sociotechnical arrangements. Simply stated, this approach to synthetic subjectivity allows for consideration of how compositions of partial objects (eyes, hands, remote controls, cables, servers, etc.) are connected, inscribed, and recognized as subject. Streaming media is driven by data that, while often examined in a purely quantifiable sense, bears historical traces that are both human and non-human. Blackman's (2019) hauntology of data allows attention to how data is shaped and reshaped by the experience of user, which allows for confrontation of "specters, displacements, disjointed times, submerged events, and multiple temporalities" (p. 20). Taken together, the conjunction of hauntology and synthetic subjectivation of streaming media allows examination of streaming subject untethered from reliance on living human bodies.

Accordingly, it is possible to conceptualize the streaming subject as more than living human beings watching, recording, uploading, and liking audio-visual content. One of the aphorisms of streaming data collection and suggestion is that platforms know the user better than they know themselves (Fiegerman, 2013). Streaming media channels a desire for personalized entertainment-through data-driven mechanisms, the user is imbricated into the machines of connection, recording, coding, production, and suggestion. The streaming subject certainly can be an arrangement that includes a living human individual who is expected to engage in cycles of content viewingrecording-rating and accompanying data collection. However, when accounting for the presence of haunted data, the streaming subject is no longer reliant on a body of flesh and blood, but can instead operate as an entanglement of machines, servers, datapoints, software, infrastructures, and algorithms that work toward the suggestion, creation, and engagement with streaming content. From this perspective, streaming and social media "do not inherently rely on human subjects, but rather derive economic value from anything that can maintain connections and circulations-which, 
consequently reduces the subject of social media to data itself" (Bollmer, 2016, p. 133). This datafied subject, accordingly, is not delimited to the boundaries of the living, but instead functions as an amalgamation of data haunted by traces of human, material, and technical histories. More simply stated, while streaming media does produce sociotechnical subjects as bounded human bodies connected to audio-visual content and platform infrastructures, is it is equally important to consider the possibility of subjectivity where the human is a mere figure in the sand (Wiley \& Elam, 2018)—where the hominid component is absent entirely, or reduced to haunted data that transcends corporeal life and death.

\section{References}

Althusser, L. (1971). Ideology and ideological state apparatuses (notes towards an investigation). New York: Verso.

Blackman, L. (2019). Haunted data: affect, transmedia, weird science. London; New York: Bloomsbury Academic.

Bollmer, G. (2016). Inhuman networks: social media and the archaeology of connection. New York: Bloomsbury Academic.

Deleuze, G., and Guattari, F. (1983) [1972]. Anti-oedipus: capitalism and schizophrenia. Minneapolis: University of Minnesota Press.

Esposti, S. D. (2014). When big data meets dataveillance: the hidden side of analytics. Surveillance \& Society, 12(2), 209-225.

Fiegerman, S. (2013). Netflix's Data Points Are Not the Usual Suspects. Retrieved from https://mashable.com/2013/12/11/netflix-data/

Foucault, M. (1969). The archaeology of knowledge. New York: Pantheon.

Fuller, M. (2009). Active Data and its Afterlives. Retrieved from

http://fuller.spc.org/fuller/matthew-fuller-active-data-and-its-afterlives/

Öhman, C. J., \& Watson, D. (2019). Are the dead taking over Facebook? A Big Data approach to the future of death online. Big Data \& Society, 6(1), 1-13. 
Saltzman, M. (2018). How to see everything Netflix knows about you. Retrieved from https://www.usatoday.com/story/tech/columnist/saltzman/2018/04/17/you-can-see-whatnetflix-knows-you-but-you-cant-download/510782002/

Spilker, H., Ask, K., \& Hansen, M. (2018). The new practices and infrastructures of participation: how the popularity of Twitch.tv challenges old and new ideas about television viewing. Information, Communication \& Society, 1-16.

Wiley, S., \& Elam, J. (2018). Synthetic subjectivation: technical media and the composition of posthuman subjects. Subjectivity, 11(3), 203-227.

Wright, N. (2014). Death and the Internet: The implications of the digital afterlife. First Monday, 19(6).

\section{GRIEF BY THE BYTE: CONSTRUCTIONS OF DATA CONSENT, PRIVACY, AND STABILITY IN GRIEFBOTS}

Tyler DeAtley

North Carolina State University

This presentation seeks to examine the phenomenon of chatbots created from a deceased person's data called griefbots. These griefbots are programmed by compiling an individual's digital footprint from social media content, text messages, and emails to create an artificial intelligence that replicates the deceased's interaction patterns to help friends and family to work through the grieving process (Villaronga, 2019). Griefbots are enabled by the vast amounts of data and content referred to as digital remains; with a minimum of 1.4 billion profiles left behind on FB alone by 2100 , there will be plenty of remains to draw from (Lingel, 2013; Öhman and Watson, 2019). As Whitt (2017) notes, however, our data, much like our own material bodies, is impermanent-the material infrastructure that houses that data can breakdown, degrade, or be wiped. This data fragility contrasts to the way that algorithms are often positioned by the companies that utilize them. Companies such as Netflix routinely articulate algorithms as a computational process that knows us better than we know ourselves (Fiegerman, 2013). Moreover, due to the computational nature of programming language, algorithms appear efficient, "impartial, reliable and legitimate" (Kitchin, 2017, pg. 19). That data is situated as objective and reliable obscures its ephemeral nature; in actuality, this data is deeply contextual, contingent, and unstable.

It is precisely this contextuality that is at the heart of griefbots. As Newton (2016) says in their essay regarding a friend's programed griefbot:

You may feel less comfortable with the idea of your texts serving as the basis for a bot in the afterlife - particularly if you are unable to review all the texts and social media posts beforehand. We present different aspects of ourselves to 
different people, and after infusing a bot with all of your digital interactions, your loved ones may see sides of you that you never intended to reveal.

In life users create messages and content with specific contexts and imagined audiences in mind (Marwick and boyd, 2011). Social media affordances such as searchability and indexability collapse those varying contexts across different temporalities. After death, when a user's data is used to create a griefbot, that context collapses completely and is presented as a whole entity, user and audience wrapped together. Moreover, the contingent and contextual aspect of the data used to create griefbots complicates the consent of both user and audience.

How programmers reconcile and understand both context and consent when programing griefbots, as well as how platforms negotiate the ethics of this data use when creating terms of use agreements warrants further scholarly attention. This presentation, therefore, seeks to interrogate the data practices surrounding the creation and implementation of griefbots' relationship to consent and privacy. For example, the data used to create griefbots comes both from the user generating the griefbot, and also from the other platform users with whom the original user interacts. Likewise, griefbots programing is being refined to incorporate learning capabilities and creating bots that have capacities to be socially active and give off appearances of sentience (Basset, 2018; Savin-Baden and Burden, 2019). The data generated by grieving users through interaction with the griefbot will be factored into those learning capabilities and used to refine the artificial intelligence. At question then is what measures are taken to protect the data that is algorithmized to create the griefbot along with how programmers understand and gather consent with the data produced from grievers and mourners' interactions with the griefbot.

In analyzing issues of consent, privacy, stability, and impermanence of griefbots, I turn to terms of use agreements, digital editorials, and existing interviews with griefbot programmers. I seek to understand how programmers conceptualize and put into practice concerns of consent, privacy, and data stability. As Kitchin (2017) argues for and Mager (2015) demonstrates, interviewing algorithm programmers is essential to understanding knowledge production practices within algorithmic industries. Through an examination of industry knowledge practices, we can understand how values such as consent and data stability are understood internally and instantiated into policy. Scholarly literature has not addressed the way griefbot programmers understand consent and privacy through a qualitative practice such as interviewing, in turn presenting a potential fruitful gap to better ascertain the formulation of griefbots. Consequently, I seek to answer how programmers see individual griefbots as stable and long-lasting, akin to digital graveyards (Nansen et al, 2014). In doing so, this presentation responds to the troubling epistemology of algorithms as objective and stable through attention to how programmers understand and implement knowledge practices around consent/privacy and data stability. If the goal is to create therapeutic griefbots that assist grieving process as much as possible while maintaining an ethics that respects the consent and privacy of all involved, it matters that programmers understand the contextual and fragile nature of the data used to generate griefbots. If not griefbots may be seen as 'true' reflections of that person, which creates a host of different problematics if consent and privacy are not respected. 


\section{References}

Bassett, D. (2018). "Digital Afterlives: From Social Media Platforms to Thanabots and Beyond" in Death and Anti-death: two hundred years after Frankenstein. (C. Tandy, Ed.) (Vol. 16). S.I.: Ria University Press.

Fiegerman, S. (2013). Netflix's Data Points Are Not the Usual Suspects. Retrieved from https://mashable.com/2013/12/11/netflix-data/

Kitchin, R. (2017). Thinking critically about and researching algorithms. Information, Communication \& Society, 20(1), 14-29.

Leaver, T. (2013). The social media contradiction: Data mining and digital death. $M / C$ Journal: A Journal of Media and Culture, 16(2).

Lingel, J. (2013). The digital remains: Social media and practices of online grief. The Information Society, 29(3), 190-195.

Marwick, A. E., \& boyd, D. (2011). I tweet honestly, I tweet passionately: Twitter users, context collapse, and the imagined audience. New media \& society, 13(1), 114-133.

Mager, Astrid. "Algorithmic Ideology: How Capitalist Society Shapes Search Engines." Information, Communication \& Society 15, no. 5 (April 10, 2012): 769-87. Accessed December 8, 2017. doi:10.1080/1369118X.2012.676056

Nansen, B., Arnold, M., Gibbs, M., \& Kohn, T. (2014). The restless dead in the digital cemetery. Digital death: Mortality and beyond in the online age, 111-124.

Newton, C. (2016, October 6). Speak, Memory. Retrieved from https://www.theverge.com/a/luka-artificial-intelligence-memorial-roman-mazurenko-bot

Öhman, C. J., \& Watson, D. (2019). Are the dead taking over Facebook? A Big Data approach to the future of death online. Big Data \& Society, 6(1), 2053951719842540.

Savin-Baden, M., \& Burden, D. (2019). Digital immortality and virtual humans. Postdigital Science and Education, 1(1), 87-103. 
Villaronga, E. F. (2019). "I Love You," Said the Robot: Boundaries of the Use of Emotions in Human-Robot Interactions. In Emotional Design in Human-Robot Interaction (pp. 93-110). Springer, Cham.

Whitt, R. S. (2016). Through a glass, darkly: Technical, policy, and financial actions to avert the coming digital dark ages. Santa Clara Computer \& High Tech. LJ, 33, 117229.

\section{WHAT IS DEAD MAY NEVER DIE: THE COMMODIFICATION OF DEATH IN SOCIAL MEDIA}

Jeffery Bruinsma

North Carolina State University

The question of what happens to our data after we die is both exigent and complex. Scholarship ranges from social media grieving practices (Pennington 2011), to the effects of memory and digital existence after death (Wright 2014; Bollmer 2016), to the political and ethical concerns surrounding the continued engagement with dead user's data (Öhman and Floridi 2018; Karppi 2018; Öhman and Watson 2019). Grief and memorialization are important emergent aspects of social media use-the empathetic drive to sustain our connection to lost loved ones is a powerful incentive for platforms like Facebook to implement regulations and policies concerning the sustained interaction of deceased user accounts. However well-intentioned various companies may be in respect to the dead, the fact remains that these are platforms storing, exchanging, and selling vast amounts of data. As Karppi (2018) argues about the transition of live accounts after death, "the conversion of dead user profiles into memorial accounts 'thingifies' the deceased, and when user profiles become things, they have not only personal or cultural value but also use-value and exchange-value" (p. 101). One of the ways in which thingification occurs is reflected in many platforms' policies concerning memorialized accounts, including Facebook and Twitter, that insist on preserving the deceased's presence while simultaneously allowing living users to continue to engage with the profile. Considering the revelations of recent data breaches, the concerns about what happens with our data while we are still living may seem more pressing than the implications of what may happen with our data after we are gone. Yet, the commodification of the dead has substantial implications not only for the shifting boundaries of life and death via big data, but also to notions of digital labor and the accompanying ethics surrounding data use. Following Terranova (2004) on the digital free labor economy and Dyer-Witheford's (2015) conceptualization of cyber class construction, including its connections and limitations across the global economy, I will discuss the interest social media platforms have in storing, maintaining, and encouraging continuous engagement with dead user profiles and data. By contextualizing these burgeoning strategies of commodification, I examine the material conditions of control and surveillance to the theoretical network conditions that produce hegemonic influences and data capital from the dead. 
At the heart of this emergent power relation are theories of the commodification and exploitation of labor. Terranova (2004) argues that cultural and technical labor we produce in digital spaces is not "developed simply as an answer to the economic needs of capital" but rather "in relation to the expansion of the cultural industries and they are part of a process of economic experimentation with the creation of monetary value out of knowledge / culture / affect" (p. 79). Everything we do on a social media platform, from liking to posting to updating our privacy settings, is a potential commodity. Andrejevic (2007) reconceptualizes this data capture on cloud-based platforms as a digital enclosure as a digital enclosure in which user data is "increasingly detailed and fine-grained, thanks to an unprecedented ability to capture and store patterns of interaction, movement, transaction, and communication" (p. 296). The expansion of cultural practices like mourning onto social media platforms are enclosed, surveilled, and then modified in such a way that aligns with the economic interests of the platform. There is no interest in removing the data, as users feel like they should not end interaction with a dead user's profile after death (Pennington 2011). The management of these decisions is critical, as Bollmer (2016) argues that "personal information is autonomous and separate from the human body. The connection between the two is not given, but must be managed" (p. 117). Although it may be sentimentality that drives the memorialization of social network profiles, social media platform policies are instead motivated by data capture, processing, and commodification. Wright (2014) points to many ways in which dead user data is problematic, including the ownership of such data belonging either to the estate of the deceased or the hosting digital platform.

Complicating matters is that data generated by users does not remain bound to its originating platform, as seen with recent data breach scandals like Cambridge Analytica. Accordingly, even if various platforms implement policies or procedures to "retire" older legacy accounts, the amount of data in algorithmic connection after death in circulation continues. Data decay and loss are concerns that can affect the circulation of user data, but many platforms have various backups that can restore data much beyond the average lifespan of a user (Cheng 2006; Miller 2015). The data contribution from dead user accounts is not insubstantial when compared to live users and is a specific, targetable point from which platforms can profit. Social media platforms rely on advertising to fund their production and growth, and advertising in digital environments is tied near exclusively to algorithmic engagement. Algorithms do not specifically differentiate between the living and the dead and, even if they were coded to avoid dead user data, there may come a point when dead user data is the bulk of the available data online (Öhman and Watson 2019). Accounts that are not functionally contributing new data can still be engaged with accounts that are, bridging new network connections and marketing opportunities.

Consequently, what emerges is not only a new relation of power in which platforms continue to exploit dead and memorialized profiles, but something even more critical. When the dead are made to provide perpetual free labor, then the networked subject becomes a continuous member of what Dyer-Witheford (2015) calls the cyberproletariat, or "a planetary working class tasked with working itself out of a job, toiling relentlessly to develop a system of robots and networks, networked robots and robot networks, for which the human is ultimately surplus to requirements..." (p. 15). The 
development of such networks, maintained by the living but being continuously fed by the dead, is now a vested interest for digital platforms.

\section{References}

Andrejevic, M. (2007). Surveillance in the digital enclosure. The Communication Review, 10, 295-317.

Bollmer, G. (2016). Inhuman networks: social media and the archaeology of connection. New York: Bloomsbury Academic.

Cheng, J. (2006, October 19). Panasonic creates 100GB blu-ray discs to last 100 years. ArsTechnica. https://arstechnica.com/gadgets/2006/10/8032/

Dyer-Witheford, N. (2015). Cyber-proletariat: Global labour in the digital vortex. London: Pluto Press.

Karppi, T. (2018). Disconnect: Facebook's affective bonds. Minneapolis: University of Minnesota Press.

Miller, R. (2015, June 30). Inside Facebook's blu-ray cold storage data center. Data Center Frontier. https://datacenterfrontier.com/inside-facebooks-blu-ray-cold-storagedata-center/

Öhman, C. J., \& Floridi, L. (2017). The political economy of death in the age of information: A critical approach to the digital afterlife industry. Minds \& Machines, 27, 639-662.

Öhman, C. J., \& Watson, D. (2019). Are the dead taking over Facebook? A Big Data approach to the future of death online. Big Data \& Society, 6(1), 1-13.

Pennington, N. (2013). You don't de-friend the dead: An analysis of grief communication by college students through Facebook profiles. Death Studies, 37, 617-635.

Terranova, T. (2004). Network culture: Politics for the information age. London: Pluto Press. 
Wright, N. (2014). Death and the Internet: The implications of the digital afterlife. First Monday, 19(6). 